Check for updates

Cite this: J. Mater. Chem. B, 2018, 6, 4216

Received 6th April 2018,

Accepted 18th May 2018

DOI: $10.1039 / c 8 t b 00922 h$

rsc.li/materials-b

\section{A toolbox approach for multivalent presentation of ligand-receptor recognition on a supramolecular scaffold $\dagger$}

\author{
Svenja Ehrmann, (D) $\ddagger^{\text {ab }}$ Chih-Wei Chu, (D) $\ddagger^{\mathrm{c}}$ Shalini Kumari, $\ddagger^{\mathrm{a}}$ Kim Silberreis, ${ }^{d}$ \\ Christoph Böttcher, ${ }^{b}$ Jens Dernedde, (D) ${ }^{d}$ Bart Jan Ravoo (D) *c and Rainer Haag (D) *a
}

A supramolecular toolbox approach for multivalent ligand-receptor recognition was established based on $\beta$-cyclodextrin vesicles (CDVs). A series of bifunctional ligands for CDVs was synthesised. These ligands comprise on one side adamantane, enabling the functionalisation of CDVs with these ligands, and either mannose or sulphate group moieties on the other side for biological receptor recognition. The physicochemical properties of the host-guest complexes formed by $\beta$-cyclodextrin ( $\beta$-CD) and adamantane were determined by isothermal titration calorimetry (ITC). Ligand-lectin interactions were investigated by surface plasmon resonance experiments (SPR) for the mannose ligands and the lectin Concanavalin A (ConA). Microscale thermophoresis (MST) measurements were applied for sulphatedependent binding to $\mathrm{L}$-selectin. In both cases, a multivalent affinity enhancement became apparent when the ligands were presented on the CDV scaffold. Furthermore, not only the clustering between our supramolecular mannosylated complex and Escherichia coli (E. coli), expressing the lectin FimH, was visualised by cryo-TEM, but also the competitive character to detach bound E. coli from a cell line, representing the uroepithelial cell surface, was demonstrated. In summary, a facile and effective supramolecular toolbox was established for various ligand-receptor recognition applications.

\section{Introduction}

In biological systems, cell-linked carbohydrates generate the glycocalyx, which is involved in diverse fundamental processes, such as cell-cell recognition and downstream signalling in living organisms. ${ }^{1,2}$ The interactions, e.g., carbohydrate-lectin binding, are usually weak and non-covalent. A multivalent display of ligands and receptors on complementary surfaces, however, is a well-known concept in nature to realise higher specificity and stronger relative binding affinities. ${ }^{3}$ For instance, the adhesion of bacteria to cell surfaces requires multivalent protein-carbohydrate interactions. ${ }^{4,5}$ In particular, the adhesion

\footnotetext{
${ }^{a}$ Institute for Chemistry and Biochemistry, Freie Universität Berlin, Takustr. 3, 14195 Berlin, Germany. E-mail: haag@chemie.fu-berlin.de

${ }^{b}$ Research Center of Electron Microscopy, Freie Universität Berlin, Fabeckstr. 36a, 14195 Berlin, Germany

${ }^{c}$ Organic Chemistry Institute and Center for Soft Nanoscience, Westfälische Wilhelms-Universität Münster, Corrensstr. 40, 48149 Münster, Germany. E-mail: b.j.ravoo@uni-muenster.de

${ }^{d}$ Charité-Universitätsmedizin Berlin, Corporate member of Freie Universität Berlin, Humboldt-Universität zu Berlin, Berlin Institute of Health, Institute of Laboratory Medicine, Clinical Chemistry and Pathobiochemistry, CVK Augustenburger Platz 1, 13353 Berlin, Germany

$\dagger$ Electronic supplementary information (ESI) available. See DOI: 10.1039/c8tb00922h

\$ The authors contributed equally.
}

of uropathogenic E. coli (UPEC) to uroepithelial cells in the urinary tract is mediated by the binding of $\alpha$-D-mannosides, presented on cell surface proteins, to the bacterial lectin FimH of type I pili of UPECs. ${ }^{6}$ In this specific case, urinary tract infections (UTI) are mostly caused by antibiotic resistant $E$. coli strains ${ }^{7-9}$ and therefore alternative treatment options are badly needed.

To achieve an effective inhibition of bacterial colonisation of tissues, synthetic multivalent scaffolds as binding competitors are a promising option. Different variables including valency, topology, density and cluster effects of carbohydrates at synthetic scaffolds are currently under investigation. ${ }^{10-14}$ Biomimetic materials have obtained increasing interest in this regard in recent years. Glycoconjugates and supramolecular self-assembled glycoclusters have been used to generate multivalent systems, ${ }^{15}$ including liposomes, ${ }^{16}$ glycopeptides, ${ }^{17}$ dendrimersomes, ${ }^{18}$ and fluorescent glycoprobes. ${ }^{19}$ The development of a toolbox system, mimicking the glycocalyx and being easily variable by adapting different suitable ligands is therefore of high interest.

Bilayer vesicles are dynamic supramolecular structures that can be used as mimic for the glycocalyx by functionalising the surface of the vesicles with carbohydrates. ${ }^{20}$ Cyclodextrins are cyclic oligosaccharides, containing D-glucopyranose as repeating units. The special orientation of glucopyranose units in cyclodextrins shapes a conical structure with a hydrophobic cavity. In addition, the 
hydroxyl groups on both sides at the open ring allows for further functionalization. $^{21}$ By amphiphilic functionalisation with alkyl chains and oligo(ethylene glycol) chains, the formation of bilayer vesicles was reported. ${ }^{22}$ Depending on the number of glucopyranose units ( 6 for $\alpha$-, 7 for $\beta$ - and 8 for $\gamma$-cyclodextrins), the size of the hydrophobic cavity ranges from 5.7 to $9.5 \AA$, thus enabling the formation of inclusion complexes with different hydrophobic guest molecules in water. ${ }^{23}$ For $\beta$-CD, adamantane is known to be a wellfitting inclusion guest $\left(K_{\mathrm{a}} \sim 10^{4} \mathrm{M}^{-1}\right)$. By choosing different guest conjugates, the self-assembled cyclodextrin vesicles (CDV) are versatile scaffolds for various applications. ${ }^{24-26}$ A similar hostguest system based on amphiphilic cucurbit[6]uril and spermidine derivatives was reported for Concanavalin A (ConA) recognition ${ }^{27}$ and photoresponsive capture and release of lectins could be achieved by introducing azobenzene motifs ${ }^{28}$ into the ligand structure.

The enhanced development of a toolbox scaffold system that enables diverse variation of its ligands and the investigation of subsequent ligand recognition were the aims of this work. The versatility concept was demonstrated by a few examples. Therefore, CDVs were non-covalently functionalised with two different kinds of lectin-recognising ligands. Mannose-presenting ligands were chosen for the binding to ConA and FimH expressing E. coli, whereas sulphate-presenting ligands were used to show L-selectin binding. ConA is a plant lectin, homotetrameric at neutral $\mathrm{pH}$ and has four carbohydrate recognition domains for mannosides. L-selectin is an adhesion protein that supports the attachment of leukocytes to endothelial cells during the inflammation process. ${ }^{29-32}$ PSGL-1 is one of the physiological ligands for L-selectin, bearing an anionic sulphotyrosine residue, which binds to the positively charged region of L-selectin. ${ }^{33,34}$ Due to this electrostatic interaction, sulphated ligands with a high local anionic charge density have been reported previously for the effective binding to L-selectin ${ }^{35}$ including derivatives of heparin ${ }^{36}$ and dendritic polyglycerol sulphates ${ }^{37}$ (dPGS), which were blocking the interaction between leukocytes and endothelial cells.

To enhance the binding affinity of ligands towards the CDV surface, also divalent adamantane anchoring moieties were considered. ${ }^{38,39}$ In addition, we synthesised ligands with different amounts of mannosides and sulphate groups, respectively, to investigate the influence of ligand density on CDVs in terms of target recognition. Therefore, interactions with the desired lectins were investigated by surface plasmon resonance (SPR) and microscale thermophoresis experiments (MST) as well as turbidimetric analysis of rising aggregates $\left(\mathrm{OD}_{400}\right)$. Furthermore, all host-guest complexes were analysed regarding their physicochemical properties by ITC measurements. In a second step, binding of mannosedecorated CDVs to FimH expressing E. coli was investigated by cryo-TEM experiments and studied in a competitive binding assay to the uroepithelial cell line RT4.

\section{Results and discussion}

\section{Synthesis}

In order to explore the multivalent supramolecular binding of cyclodextrin vesicles (CDVs), seven different ligands were

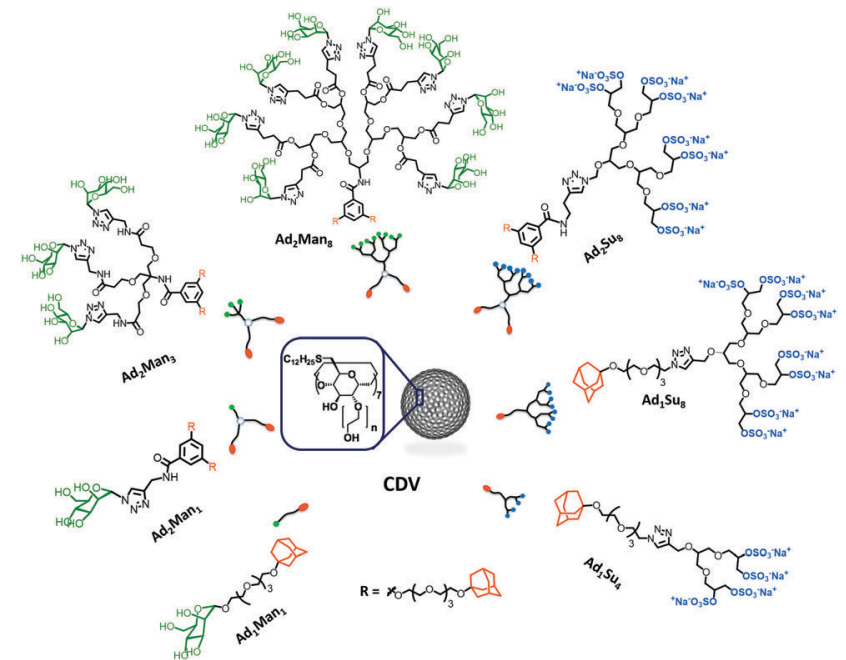

Scheme 1 Schematic representation of the synthesised adamantyl ligands with mannose and sulphate motifs and amphiphilic cyclodextrin vesicles.

synthesised, four of them bearing mannosides and three with sulphate group functionalisation. The valency of the ligands included mono-, tri- and octavalent mannosides as well as tetraand octavalent sulphates in order to study the effect of multivalent ligand-receptor binding. The ligands were designed so that on one side of the structure a motif of one or two adamantyl residues allowed for the complexation to $\beta$-cyclodextrins ( $\beta$-CD) at the surface of CDVs. Adamantyl groups are known to form inclusion complexes with $\beta$-CD with an affinity constant of around $K_{\mathrm{a}} \sim 10^{4} \mathrm{M}^{-1}$ for monovalent 1:1 binding. Divalent binding with two adamantane residues per ligand, however, can lead to an apparent affinity constant of up to $K_{\mathrm{a}} \sim 10^{7} \mathrm{M}^{-1}$ plus additional kinetic stabilization of the complex. ${ }^{40}$ On the opposite side of the ligands, either mannose or sulphate groups were introduced. The synthetic ligands are shown in Scheme 1 and their detailed synthesis procedures are described in the ESI. $\dagger$

The octavalent mannoside ligand $\mathbf{A d}_{\mathbf{2}} \mathbf{M a n} \mathbf{n}_{\mathbf{8}}$ was synthesised as shown schematically in Fig. S4 (ESI $\dagger$ ). A polyglycerol-based dendron was synthesised according to published procedures ${ }^{41}$ and coupled to a two-adamantane precursor by amide bond formation. Afterwards, the acetal-protection groups of the dendron were cleaved off using Dowex ${ }^{\circledR}$ resin. The free hydroxyl groups were then converted into terminal alkyne groups by the addition of 4-pentynoic acid via esterification. Subsequently, azido-mannosides were clicked onto the dendron's terminal alkyne groups via copper-catalysed azide-alkyne cycloaddition (CuAAC) under optimised conditions. The reaction was stirred for four days at elevated temperature $\left(50{ }^{\circ} \mathrm{C}\right)$. The addition of a piece of copper wire ${ }^{42}$ along with $\mathrm{CuSO}_{4}$ and sodium ascorbate turned out to facilitate the reaction drastically and led to full octavalent functionalisation. Sulphate-adamantyl conjugates were synthesised in a similar procedure. Here, the terminal hydroxyl groups were sulphated using a sulphur trioxide pyridine complex.

Amphiphilic $\beta$-cyclodextrins functionalised with alkyl and polyethylene glycol chains were synthesised according to 
previous reports. ${ }^{24} \beta$-Cyclodextrin vesicles (CDV) were obtained by extrusion, yielding vesicles with diameters between 100 and $200 \mathrm{~nm}$.

\section{Physicochemical characterisation}

In order to investigate the vesicle's size and morphology, dynamic light scattering (DLS) and cryogenic transmission electron microscopy (cryo-TEM) experiments were carried out.

Cryo-TEM images show mono- and multilayered vesicles with diameters of $(177 \pm 40) \mathrm{nm}$ (Fig. 1A). These values closely correlate with the range of 140 to $180 \mathrm{~nm}$ obtained by DLS measurements (Fig. 1D), regardless of the solvent used (Milli-Q, HEPES, PBS+/+). Even after addition of mannoside ligands, the shape and size of the vesicles remained similar as can be seen in Fig. $1 \mathrm{~B}$ and $\mathrm{C}$.

The host-guest interaction was investigated between adamantane mannoside ligands $\left(\mathbf{A d}_{\mathbf{1}} \mathbf{M a n}_{1}, \mathbf{A d}_{\mathbf{2}} \mathbf{M a n}_{\mathbf{1}}, \mathbf{A d}_{\mathbf{2}} \mathbf{M a n}_{3}\right.$, and $\left.\mathbf{A d}_{\mathbf{2}} \mathbf{M a n}_{\mathbf{8}}\right)$ and $\beta-\mathrm{CD}$ as non-aggregated $\beta-\mathrm{CD}$ allows unhindered inclusion complex formation with adamantane. Measurements were carried out by isothermal titration calorimetry (ITC) using $1: 1$ complexes of adamantane and $\beta$-CD (Table 1). Each titration was performed with a 10-fold higher host concentration in relation to the guest molecule. All ligands showed association constants in the range of $10^{4} \mathrm{M}^{-1}$, which are in the typical range of adamantane to $\beta$-CD binding and are in line with previous results. ${ }^{26}$ The thermodynamic parameters of $\mathbf{A d}_{\mathbf{1}} \mathbf{M a n}_{\mathbf{1}}, \mathbf{A d}_{\mathbf{2}} \mathbf{M a n}$, and $\mathbf{A d}_{2} \mathbf{M a n}_{\mathbf{3}}$ are consistent (negative $\Delta H$ and positive $\Delta S$ ), while for $\mathbf{A d}_{\mathbf{2}} \mathbf{M a n}_{\mathbf{8}}$ the change in entropy $(\Delta S)$ is slightly negative and the change in enthalpy $(\Delta H)$ is slightly increased. We attribute this behaviour to the bulky and strongly hydrated
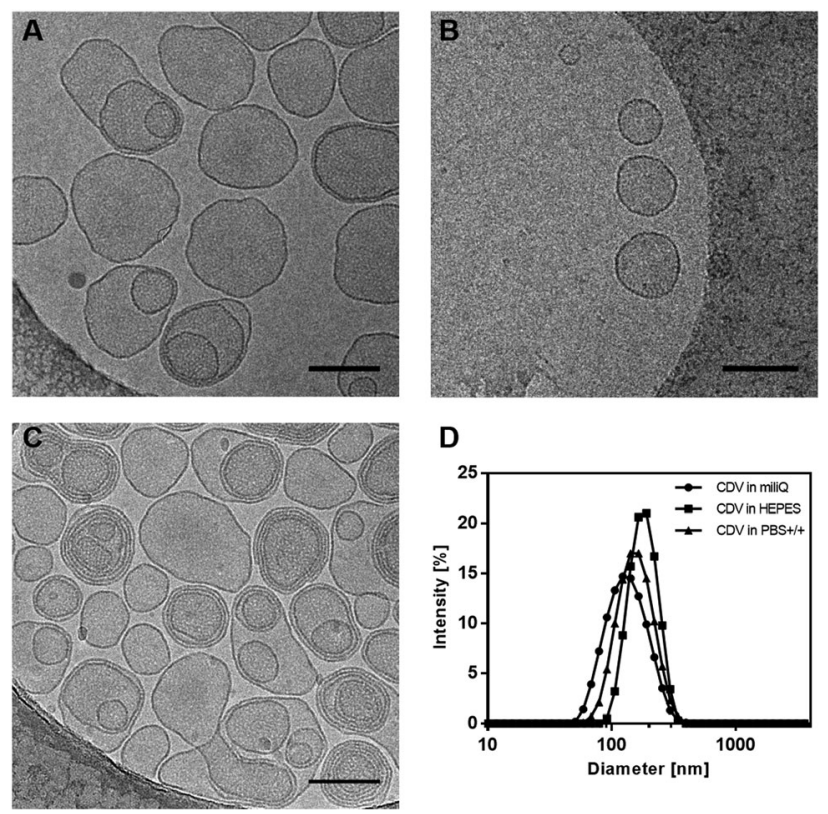

Fig. 1 Cryogenic transmission electron microscopy of CDVs in water: (A) $1 \mathrm{mMCDV}$; (B) $100 \mu \mathrm{M} \mathrm{CDV}+25 \mu \mathrm{M} \mathrm{Ad}_{2}$ Man $_{1}$; (C) $100 \mu \mathrm{M} \mathrm{CDV} \mathrm{+} 25 \mu \mathrm{M}$ $\mathrm{Ad}_{\mathbf{2}} \mathbf{M a n}_{\mathbf{8}}$. Scale bars represent $150 \mathrm{~nm}$ in all figures. The displayed cryoTEM images are representative and show uni- and multilamellar vesicles as well as their structural stability even after the addition of mannoside ligands. (D) Size distribution of CDVs in different aqueous media measured by dynamic light scattering.
Table 1 Summary of physicochemical properties for adamantanemannose ligand complexes determined by ITC

\begin{tabular}{lllll}
\hline $\begin{array}{l}\text { Ligand } \\
\text { at CDV }\end{array}$ & $\begin{array}{l}\Delta H \\
\left.(\mathrm{~kJ} \mathrm{~mol})^{-1}\right)\end{array}$ & $\begin{array}{l}\Delta G \\
(\mathrm{~kJ} \mathrm{~mol}\end{array}$ & $\begin{array}{l}\Delta S \\
\left(\mathrm{~J} \mathrm{~K}^{-1} \mathrm{~mol}^{-1}\right)\end{array}$ & $K_{\mathrm{a}}\left(\mathrm{M}^{-1}\right)$ \\
\hline $\mathbf{A d}_{\mathbf{1}} \mathbf{M a n}_{1}$ & -16.7 & -26.0 & 31.0 & $3.55 \times 10^{4}$ \\
$\mathbf{A d}_{2} \mathbf{M a n}_{1}$ & -18.1 & -26.3 & 17.6 & $1.24 \times 10^{4}$ \\
$\mathbf{A d}_{2} \mathbf{M a n}_{3}$ & -18.4 & -23.6 & 17.5 & $1.38 \times 10^{4}$ \\
$\mathbf{A d}_{2} \mathbf{M a n}_{8}$ & -25.2 & -23.3 & -6.1 & $1.23 \times 10^{4}$ \\
\hline
\end{tabular}

octavalent mannose dendron, which will significantly reduce the entropic bonus of releasing hydration water from the adamantane. Instead, it will be first solvated by the dendron and then included by the $\beta-\mathrm{CD}$, so that the net gain in entropy due to the desolvation is minimal or even negative. In addition, $\Delta H$ is higher assumingly because of multiple weak but favourable hydrogen bonds between the dendron and the $\beta$-CD. However, the resulting association constant $K_{\mathrm{a}}$ lies within the same range of the other ligands, which results in a stable inclusion complex formation for all ligands. Furthermore, due to the divalent binding of the ligands $\mathbf{A d}_{2} \mathbf{M a n}_{1}, \mathbf{A d}_{2} \mathbf{M a n}_{3}$ and $\mathbf{A d}_{\mathbf{2}} \mathbf{M a n}_{\mathbf{8}}$ to the membrane of CDVs, increased binding affinities $\left(K_{\mathrm{a}} \sim 10^{7} \mathrm{M}^{-1}\right)$ and additional stabilization effects for the complexes can be assumed as shown by Huskens et al. ${ }^{39}$ Additional titration plots including fitting curves are given in ESI, $\dagger$ (Fig. S5-S7).

In a second set of experiments, the non-covalent interactions between $\beta-C D$ and the sulphated adamantyl ligands were investigated by ITC. Additionally, the non-sulphated analogues (OH-group terminated) were included in this assay to examine the effect of sulphation on the thermodynamic parameters. Again, reasonable binding affinities $\left(K_{\mathrm{a}} \sim 10^{4} \mathrm{M}^{-1}\right)$ for adamantane to $\beta$-CD were observed for all ligands (Table 2). In the case of $\mathbf{A d}_{\mathbf{2}} \mathbf{O H}_{\mathbf{8}}$, a binding affinity comparable to the mannosefunctionalised ligands ( $c f$. Table 1) was obtained. A similar effect as before could be observed, i.e., a negative change in entropy and an increased change in enthalpy, which we again attribute to the strongly hydrated and bulky octavalent dendron in combination with two adamantane moieties. By comparing the titrations of $\mathbf{A d}_{\mathbf{2}} \mathbf{O H}_{\mathbf{8}}$ and $\mathbf{A d}_{\mathbf{2}} \mathbf{S} \mathbf{u}_{\mathbf{8}}$, an unexpected behaviour was observed, i.e., the sulphated ligand showed not only a lower heat rate change by a factor of five than $\mathbf{A d}_{\mathbf{2}} \mathbf{O} \mathbf{H}_{\mathbf{8}}$, but also reached the thermal equilibrium already after three titrations (Fig. S7, ESI $\dagger$ ). This observation suggests that a self-assembled structure of $\mathbf{A d}_{2} \mathbf{S u}_{\mathbf{8}}$ was present as the amphiphilicity of $\mathbf{A d}_{\mathbf{2}} \mathbf{S u}_{\mathbf{8}}$ increased due to the sulphation of the highly branched headgroup in comparison to the uncharged $\mathbf{A d}_{\mathbf{2}} \mathbf{O} \mathbf{H}_{\mathbf{8}}$. Thus, the

Table 2 Summary of physicochemical properties for adamantane-

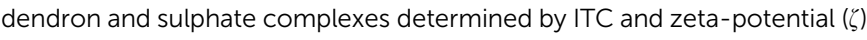

\begin{tabular}{lllllr}
\hline $\begin{array}{l}\text { Ligand } \\
\text { at CDV }\end{array}$ & $\begin{array}{l}\Delta H \\
\left(\mathrm{~kJ} \mathrm{~mol} \mathrm{~mol}^{-1}\right)\end{array}$ & $\begin{array}{l}\Delta G \\
\left(\mathrm{~kJ} \mathrm{~mol}{ }^{-1}\right)\end{array}$ & $\begin{array}{l}\Delta S \\
\left(\mathrm{~J} \mathrm{~K}^{-1} \mathrm{~mol}^{-1}\right)\end{array}$ & $K_{\mathrm{a}}\left(\mathrm{M}^{-1}\right)$ & $\zeta(\mathrm{mV})$ \\
\hline$\beta-\mathrm{CDV}$ & - & - & - & - & $-10.0 \pm 1.7$ \\
$\mathbf{A d}_{\mathbf{1}} \mathbf{O} \mathbf{H}_{4}$ & -17.4 & -25.9 & 28.5 & $3.45 \times 10^{4}$ & $-9.5 \pm 0.4$ \\
$\mathbf{A d}_{\mathbf{1}} \mathbf{S u}_{4}$ & -16.8 & -25.2 & 28.1 & $2.59 \times 10^{4}$ & $-14.6 \pm 1.1$ \\
$\mathbf{A d}_{\mathbf{1}} \mathbf{O H}_{\mathbf{8}}$ & -18.0 & -25.1 & 23.6 & $2.46 \times 10^{4}$ & $-8.5 \pm 0.9$ \\
$\mathbf{A d}_{\mathbf{1}} \mathbf{S u}_{8}-17.3$ & -24.1 & 22.6 & $1.66 \times 10^{4}$ & $-16.3 \pm 2.4$ \\
$\mathbf{A d}_{\mathbf{2}} \mathbf{O H}_{\mathbf{8}}-25.9$ & -23.6 & -7.6 & $1.38 \times 10^{4}$ & $-5.3 \pm 0.2$
\end{tabular}


adamantane groups were hindered from binding to $\beta$-CD. For all other ligands, this behaviour was not observed.

Zeta-potential measurements were carried out to investigate the charge of CDVs after the complexation with neutral and sulphated ligands at the CDV surface (Table 2). For the dendritic neutral ligands, $\mathbf{A d}_{\mathbf{1}} \mathbf{O H}_{\mathbf{4}}$ and $\mathbf{A d}_{\mathbf{1}} \mathbf{O H}_{\mathbf{8}}$, the zeta-potential remained unchanged after addition of the ligands in comparison to the bare CDVs $(\zeta=-8-10 \mathrm{mV})$, which also contain ethylene glycol side chains at the vesicle surface.

In contrast, the decoration of CDVs with the sulphated ligands $\mathbf{A d}_{\mathbf{1}} \mathbf{S u}_{\mathbf{4}}$ and $\mathbf{A d}_{\mathbf{1}} \mathbf{S u}_{\mathbf{8}}$ led to an increased negative zetapotential $(\zeta=-14-16 \mathrm{mV}$ ), as the negatively charged ligands changed the surface environment after their complexation to CDVs.

In the following, supramolecular complexes, consisting of CDVs and mannose-functionalised or sulphated adamantyl ligands, respectively, were investigated regarding their binding affinities towards lectins.

\section{Lectin binding}

Surface plasmon resonance (SPR). SPR were performed to analyse the binding affinities of mannose-functionalised CDVs to the lectin Concanavalin A (ConA). ConA originates from the jack bean Canavalia ensiformis and forms homotetramers above $\mathrm{pH} 7$ and in the presence of $\mathrm{Ca}^{2+}$ as well as $\mathrm{Mn}^{2+}$ ions. ${ }^{1}$ The carbohydrate recognition site is specific for mannose and glucose, which has turned ConA into a well-studied model system for mannose-binding.

In this assay, the aim was to compare binding affinities between the synthetic mannose-functionalised ligands as well as the respective ligand-functionalised vesicles to ConA. Therefore, ConA-biotin was immobilised on a streptavidin-functionalised SPR sensor chip, whereas mannose ligands and ligand-functionalised vesicles, dissolved in HEPES buffer, were run in a continuous flow over the sensor chip (Fig. 2A). In a kinetic titration series, binding
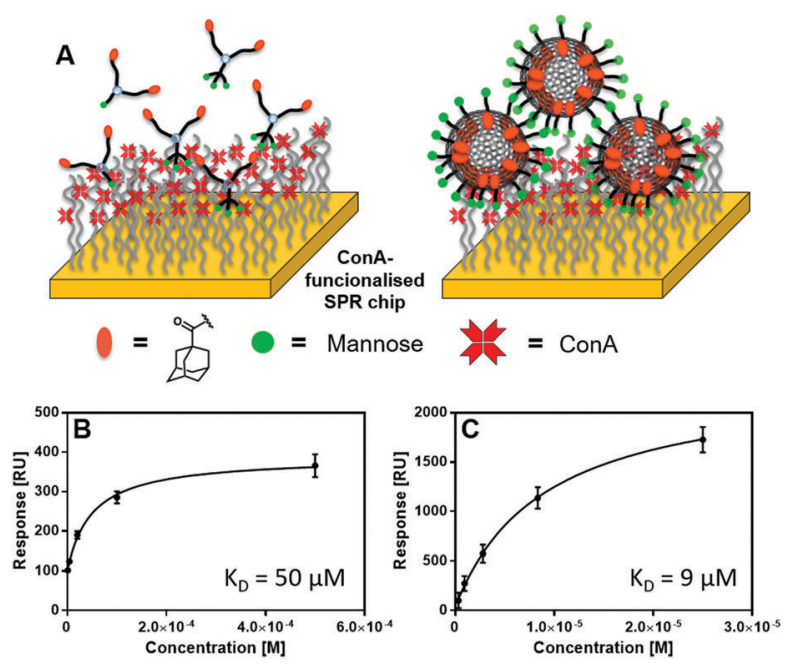

Fig. 2 (A) Schematic depiction of SPR experiment setup. (B) Determination of binding affinities for mannoside functionalised ligands (left) as well as ligand functionalised vesicles (right) to ConA. (C) Corresponding binding isotherms are shown exemplary for $\mathbf{A d}_{\mathbf{2}} \mathbf{M a n}_{\mathbf{8}}$.
Table 3 Binding affinities obtained from SPR of mannose-conjugates and mannose-functionalised CDVs

\begin{tabular}{|c|c|c|}
\hline \multirow[b]{2}{*}{ Ligand } & \multicolumn{2}{|l|}{$K_{\mathrm{D}}(\mu \mathrm{M})$} \\
\hline & Bare ligand & Ligand displayed on CDVs \\
\hline $\operatorname{Ad}_{1} \operatorname{Man}_{1}$ & 234 & 89 \\
\hline $\operatorname{Ad}_{2} \operatorname{Man}_{1}$ & 50 & 7 \\
\hline $\operatorname{Ad}_{2} \operatorname{Man}_{3}$ & 69 & 13 \\
\hline $\operatorname{Ad}_{2} \operatorname{Man}_{8}$ & 50 & 9 \\
\hline
\end{tabular}

measurements were conducted with increasing concentrations of the respective sample. Subsequently, $K_{\mathrm{D}}$ values for each sample were determined by single cycle kinetics and the resulting binding isotherms (Fig. 2B, C and Fig. S10, S11, ESI $\dagger$ ). The results are summarised in Table 3.

Due to non-specific binding of dissociated adamantane to the dextran layer on the SPR chip, additional cyclodextrin was added to the SPR running buffer. In order to efficiently shield the adamantyl moiety, $\beta$-CD and $\gamma$-CD were added as surfactants to the buffer during the measurements with mannoside ligands and ligand-decorated vesicles, respectively. Further information about the experiments, showing that the system was not affected by the additives, is given in the ESI. $\dagger$

At first, binding affinities of mannose-functionalised conjugates $\left(\operatorname{Ad}_{1} \mathbf{M a n}_{1}, \operatorname{Ad}_{2} \mathbf{M a n}_{1}, \operatorname{Ad}_{2} \mathbf{M a n}_{3}\right.$ and $\left.\mathbf{A d}_{2} \mathbf{M a n}_{8}\right)$ to ConA were measured. The determined $K_{\mathrm{D}}$ values ranged from 50-234 $\mu \mathrm{M}$ (Table 3).

Subsequently, CDVs were decorated with the ligands through the formation of adamantane- $\beta-C D$ inclusion complexes. The concentrations of ligands were chosen such that full decoration of the vesicles by complexation of every cyclodextrin was theoretically enabled (referred to as $100 \%$ surface coverage). Here, determined $K_{\mathrm{D}}$ values ranged from 7-89 $\mu \mathrm{M}$. By comparison with the corresponding values for the conjugates itself, the binding was strengthened by a factor of approx. three to seven through the multivalent display of ligands at CDVs, highlighting the effectivity of multivalent organisation of ligands. However, differences in the binding affinities resulting from different carbohydrate valences (one to eight) could not be discriminated at that point.

The obtained values are comparable to recent studies also using SPR. ${ }^{43-46}$ However, in contrast to the present approach, these works used covalently synthesised glycoclusters and glycopolymers. By using a supramolecular multivalent approach, a facile adaption to different targets in terms of e.g. valency and type of ligand is enabled. Additional aggregation experiments with turbidimetric measurements $\left(\mathrm{OD}_{400}\right)$ investigated the optimisation of the surface coverage of vesicles with mannoside ligands by altering the ratio of ligand to host moiety. Here, a threshold of $20 \%$ surface coverage was identified for effective aggregation for all ligands (Fig. S8 and S9, ESI $\dagger$ ). Further details of the experiments can be found in the ESI. $\dagger$

L-selectin binding analysis using label-free microscale thermophoresis (MST). In order to demonstrate the versatility of our toolbox system, the sulphated ligands and respective CDV sulphate complexes were applied in a L-selectin binding assay. Here, the dissociation constant was determined by microscale 

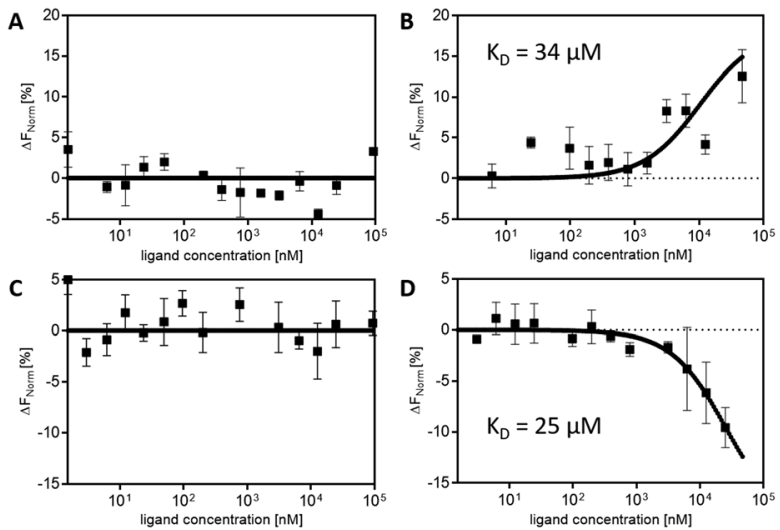

Fig. 3 MST dose-response curves of sulphated ligands $\mathbf{A d}_{1} \mathbf{S u}_{4}(A)$ without vesicles and (B) with vesicles and $\mathbf{A d}_{1} \mathbf{S u}_{8}$ (C) without vesicles and (D) with vesicles. All systems are titrated against L-selectin $(100 \mu \mathrm{M})$. Sulphated ligands without vesicles show no interaction with L-selectin, whereas in the presence of vesicles significant binding was observed. Each graph displays data merged from three independent experiments.

thermophoresis experiments (MST). MST is a powerful technique to study the interaction between proteins and small molecules in solution with low sample consumption. ${ }^{47}$ The technique makes use of molecular migration due to an applied temperature gradient. ${ }^{48}$ Binding affinities are evaluated from the changes in the thermophoretic behaviour resulting from binding of the ligand to the protein. ${ }^{49}$

The sulphated ligands $\mathbf{A d}_{\mathbf{1}} \mathbf{S u}_{\mathbf{4}}$ and $\mathbf{A d}_{\mathbf{1}} \mathbf{S u}_{\mathbf{8}}$ without CDVs showed randomly fluctuating values for the MST response throughout the ligand titration range. However, likewise to the mannose-functionalised ligands, an optimised binding behaviour due to multivalent display of the ligands on CDVs was observed. In the presence of vesicles, characteristic s-shaped and mirrored-s-shaped sigmoidal binding curves could be obtained (Fig. 3). The different appearances of the binding curves can be attributed to different thermophoretic movements of the respective ligands. ${ }^{49}$ From these experimental data, $K_{\mathrm{D}}$ values of $34 \mu \mathrm{M}$ for $\mathbf{A d}_{\mathbf{1}} \mathbf{S u}_{4}$ and $25 \mu \mathrm{M}$ for $\mathbf{A d}_{\mathbf{1}} \mathbf{S u}_{\mathbf{8}}$ were determined. These values are in the same range of those observed from mannoside-ConA binding experiments ( $c f$. SPR experiments) and underline the broad and easy applicability of non-covalently functionalised CDVs in biological binding approaches by simply changing the functionalised ligands.

In vitro assay - bacteria binding. Mannose-functionalised CDVs were further studied in a competitive in vitro assay. Here, the ability to remove bound $E$. coli bacteria (ORN178, $\mathrm{FimH}^{+}$) from the human uroepithelial cell line RT-4 was investigated. The adhesion of UPECs to uroepithelial cells, subsequent biofilm formation in the urinary system, and further bacterial invasion can lead to a severe inflammation. ${ }^{50,51}$ Strategies to prevent or disrupt the initial adhesion process are of increasing importance especially due to an increasing number of antibiotic resistant strains. ${ }^{52}$ The adhesion is mediated by the recognition and binding of the lectin FimH at the Type 1 pili of UPECs to carbohydrates at the glycocalyx of epithelial cells. FimH bears a carbohydrate recognition domain, which is specific for $\alpha$-D-mannose.
In this context, mannose-functionalised synthetic structures with high binding affinities towards FimH are highly promising antiadhesive drug candidates.

The potential of our system to detach UPECs from uroepithelial cells was investigated using an in vitro assay. Uroepithelial RT-4 cells were incubated with $E$. coli strain ORN178 for 1 h. Afterwards, mannose-functionalised vesicles were added and incubated for 15 min together with cells and bacteria. In case of successful competitive binding, the vesicles would detach the bacteria from cell surfaces. The assay principle is depicted in Fig. 4A. Subsequently, the supernatant with detached bacteria was harvested and plated in serial dilutions on agar plates. Colonies were counted after overnight incubation at $37{ }^{\circ} \mathrm{C}$ and the detaching ability was calculated as relative activity in relation to PBS buffer solution as negative control. All bi-adamantane ligands, carrying one, three and eight mannose residues were tested ( $\mathbf{A d}_{2} \mathbf{M a n}_{1}, \mathbf{A d}_{\mathbf{2}} \mathbf{M a n}_{3}, \mathbf{A d}_{\mathbf{2}} \mathbf{M a n}_{\mathbf{8}}$ ). Furthermore, ligand concentrations were chosen such that both half and full coverage of the vesicles was achieved. For comparison, also the monovalent methyl- $\alpha$-D-mannopyranoside (Me-Man) was included. The non-carbohydrate ligand Ad-TEG-OH served as a control to exclude unspecific binding. As can be seen in Fig. 4B, tri- and octavalent mannoside ligands were able to release significantly more bacteria from RT- 4 cell layers than the monovalent and control ligands. Furthermore, Me-Man and $\mathbf{A d}_{\mathbf{2}} \mathbf{M a n}_{\mathbf{1}}$ did show the same or even less detaching activity compared to the inactive control ligand Ad-TEG-OH.

In the following, different coverage densities of the vesicles with ligands were tested. As can be seen in Fig. 4B, the coverage density, i.e., half and full coverage with ligands on the CDV's surface, had no influence on the detaching efficiency. These findings seem to be non-intuitive at the first glance, i.e., one would expect a direct dependence between the detaching efficiency and ligand density. However, the above-described results can be interpreted by considering the effect of local density of binding sites. The pili of the UPECs exhibit only a single mannose-binding site, which is located at the outermost protein, i.e., FimH. At the beginning of our cell experiments, this binding site was bound to the mannosides at the uroepithelial RT4 cell membrane. By adding the functionalised vesicles, alternative mannosides were offered for binding. Since binding is a dynamic process, binding and debinding occurs occasionally. If an alternative binding site other than the natural mannosides at the cell membrane were present during debinding, FimH may bind to these alternatives. Binding to these alternatives becomes even more favourable, if they are arranged in multivalent clusters. If this is the case, they exhibit a high local concentration of binding sites. An increase of the high local concentration of binding sites leads to a decreased dissociation rate $e^{53,54}$ and statistically favoured rebinding. Hence, FimH stayed bound to the dendritic mannoside clusters. These mannoside clusters are present in the tri- and octavalent mannose ligands, explaining the increased detaching efficiency compared to the monovalent mannosides and the inactive ligand. Thus, multivalency is an important aspect for enhancing binding efficiencies in biological systems.

In addition, cryo-TEM images of UPECs and CDVs were taken (Fig. 4C-E). Here, CDVs were functionalised with either 
A

.

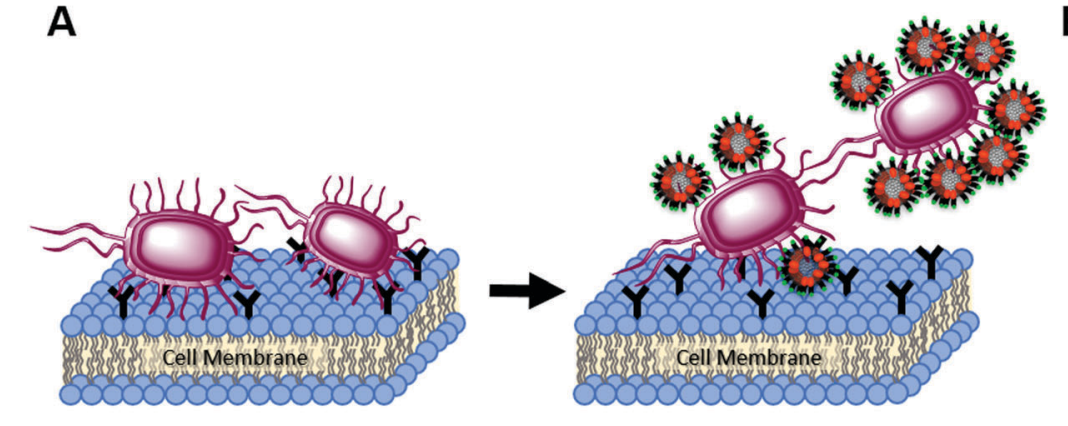

B
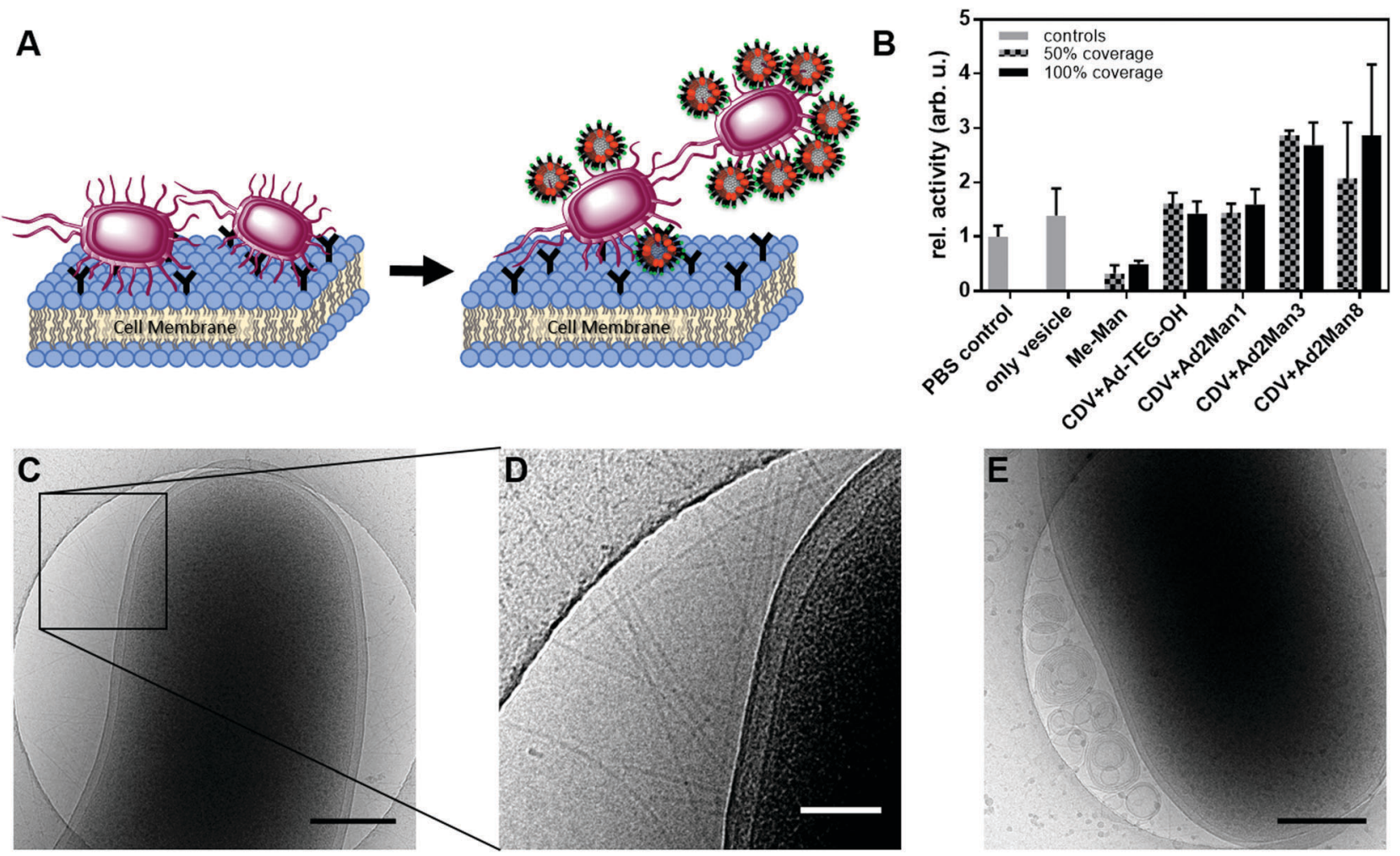

Fig. 4 (A) Schematic presentation of the bacteria-detaching assay. Left, FimH pili of E. coli bind to the mannosylated surface proteins of RT-4 cells; right, mannose-functionalised CDVs are added and competitive binding liberates $E$. coli. (B) Detaching ability of different functionalised vesicles to disturb cell-bacteria interaction. Representative cryo-TEM micrographs show mannose-dependant CDV binding to E. coli cells. (C) Control: E. coli + CDV + Ad-TEG-OH; (D) control: close-up picture of cell membrane and pili. (E) E. coli + CDV + $\mathbf{A d}_{\mathbf{2}} \mathbf{M a n} \mathbf{n}_{\mathbf{8}}$. Scale bars are $300 \mathrm{~nm}$ for (C and E) and $100 \mathrm{~nm}$ for (D).

the inactive ligand Ad-TEG-OH or the octavalent mannoside ligand $\mathbf{A d}_{\mathbf{2}} \mathbf{M a n} \mathbf{8}_{\mathbf{8}}$. For UPECs incubated with CDVs plus the inactive Ad-TEG-OH ligand, no binding of vesicles to the pili of the bacteria could be observed (Fig. 4C and D). However, vesicles, functionalised with $\mathbf{A d}_{2} \mathbf{M a n} \mathbf{8}_{\mathbf{8}}$ ligands, show clear co-localization to the bacteria and their pili (Fig. 4E), supporting the above results from the in vitro assays. Furthermore, the cryoTEM images prove that binding occurs to the functionalised vesicles and not to free and non-complexed ligands in solution.

\section{Conclusion}

In conclusion, we performed a thorough study on multivalently functionalised CDVs for lectin binding. Functionalisation of the vesicles was realised by novel carbohydrate and sulphate ligands, namely mono-, tri- and octavalent mannosides as well as tetra- and octavalent sulphates. These different ligands were used in a toolbox approach to demonstrate enhanced lectin binding through multivalent display of the ligands at the vesicle's surface. Our toolbox approach easily allowed addressing different biologically relevant receptors by modifying the supramolecular system with corresponding ligands, i.e., mannosides for ConA binding as well as sulphated ligands for L-selectin binding. Finally, we demonstrated in an in vitro assay that our mannose-functionalised vesicles are an efficient system for detaching E. coli from uroepithelial cells. FimH antagonists as anti-adhesive compounds reduce the bacterial load and therefore could be further developed for wash out strategies as a treatment option with respect to antibiotic resistant UPEC strains. In the future, new strategies to target different pathogens using the toolbox approach presented in this work will be anticipated.

\section{Conflicts of interest}

The authors declare no conflict of interests.

\section{Acknowledgements}

The authors would like to thank the German Research Foundation (DFG, SFB 765) (SE, KS, CB, JD, and RH) and the - EC H2020 Marie Skłodowska-Curie Actions - Innovative Training Network, Multi-App (project number: 642793) (CWC, SK, RH, and BJR) for financial support. The authors thank Katharina Goltsche for support in the synthesis of dendrons and Dr Christian Kühne for assisting with SPR measurements. Dr Pamela Winchester is gratefully acknowledged for language polishing the manuscript.

\section{References}

1 H. Lis and N. Sharon, Chem. Rev., 1998, 98, 637-674.

2 H. J. Gabius, S. André, J. Jiménez-Barbero, A. Romero and D. Solís, Trends Biochem. Sci., 2011, 36, 298-313. 
3 M. Mammen, S.-K. Choi and G. M. Whitesides, Angew. Chem., Int. Ed., 1998, 37, 2754-2794.

4 J. Pizarro-Cerdá and P. Cossart, Cell, 2006, 124, 715-727.

5 A. M. Krachler, H. Ham and K. Orth, Proc. Natl. Acad. Sci. U. S. A., 2011, 108, 11614-11619.

6 M. Hartmann and T. K. Lindhorst, Eur. J. Org. Chem., 2011, 3583-3609.

7 E. C. Svanborg and P. de Man, Infect. Dis. Clin. N. Am., 1987, 1, 731-750.

8 J. R. Johnson, Clin. Microbiol. Rev., 1991, 4, 80-128.

9 L. Hagberg, U. Jodal, T. K. Korhonen and C. E. Svanborg, Infect. Immun., 1981, 31, 564-574.

10 J. W. Wehner, M. Hartmann and T. K. Lindhorst, Carbohydr. Res., 2013, 371, 22-31.

11 C. Müller, G. Despras and T. K. Lindhorst, Chem. Soc. Rev., 2016, 45, 3275-3302.

12 D. Deniaud, K. Julienne and S. G. Gouin, Org. Biomol. Chem., 2011, 9, 966-979.

13 E. M. Munoz, J. Correa, E. Fernandez-Megia and R. Riguera, J. Am. Chem. Soc., 2009, 131, 17765-17767.

14 M. L. Talaga, N. Fan, A. L. Fueri, R. K. Brown, Y. M. Chabre, P. Bandyopadhyay, R. Roy and T. K. Dam, Biochemistry, 2014, 53, 4445-4454.

15 M. Delbianco, P. Bharate, S. Varela-Aramburu and P. H. Seeberger, Chem. Rev., 2016, 116, 1693-1752.

16 W. Curatolo, A. O. Yau, D. M. Small and B. Sears, Biochemistry, 1978, 17, 5740-5744.

17 J. R. Kramer, A. R. Rodriguez, U.-J. Choe, D. T. Kamei and T. J. Deming, Soft Matter, 2013, 9, 3389-3395.

18 Q. Xiao, S. S. Yadavalli, S. Zhang, S. E. Sherman, E. Fiorin, L. da Silva, D. A. Wilson, D. A. Hammer, S. André, H.-J. Gabius, M. L. Klein, M. Goulian and V. Percec, Proc. Natl. Acad. Sci. U. S. A., 2016, 113, 1134-1141.

19 K.-B. Li, N. Li, Y. Zang, G.-R. Chen, J. Li, T. D. James, X.-P. He and H. Tian, Chem. Sci., 2016, 7, 6325-6329.

20 R. V. Vico, J. Voskuhl and B. J. Ravoo, Langmuir, 2011, 27, 1391-1397.

21 B. V. K. J. Schmidt and C. Barner-Kowollik, Angew. Chem., Int. Ed., 2017, 56, 8350-8369.

22 B. J. Ravoo and R. Darcy, Angew. Chem., Int. Ed., 2000, 39, 4324-4326.

23 J. Szejtli, Chem. Rev., 1998, 98, 1743-1754.

24 P. Falvey, W. Lim, R. Darcy, T. Revermann, U. Karst, M. Giesbers, A. T. M. Marcelis, A. Lazar, A. W. Coleman, D. N. Reinhoudt and B. J. Ravoo, Chem. - Eur. J., 2005, 11, 1171-1180.

25 J. Voskuhl, M. C. A. Stuart and B. J. Ravoo, Chem. - Eur. J., 2010, 16, 2790-2796.

26 U. Kauscher and B. J. Ravoo, Beilstein J. Org. Chem., 2012, 8, 1543-1551.

27 H. K. Lee, K. M. Park, Y. J. Jeon, D. Kim, D. H. Oh, H. S. Kim, C. K. Park and K. Kim, J. Am. Chem. Soc., 2005, 127, 5006-5007.

28 A. Samanta, M. C. A. Stuart and B. J. Ravoo, J. Am. Chem. Soc., 2012, 134, 19909-19914.

29 L. M. Coussens and Z. Werb, Nature, 2002, 420, 860-867.
30 R. P. McEver, K. L. Moore and R. D. Cummings, J. Biol. Chem., 1995, 270, 11025-11028.

31 L. A. Lasky, Annu. Rev. Biochem., 1995, 64, 113-139.

32 S. D. Rosen and C. R. Bertozzi, Curr. Biol., 1996, 6, 261-264.

33 W. S. Somers, J. Tang, G. D. Shaw and R. T. Camphausen, Cell, 2000, 103, 467-479.

34 B. J. Graves, R. L. Crowther, C. Chandran, J. M. Rumberger, S. Li, K.-S. Huang, D. H. Presky, P. C. Familletti, B. A. Wolitzky and D. K. Burns, Nature, 1994, 367, 532-538.

35 E. E. Simanek, G. J. McGarvey, J. A. Jablonowski and C.-H. Wong, Chem. Rev., 1998, 98, 833-862.

36 J. Fritzsche, S. Alban, R. J. Ludwig, S. Rubant, W.-H. Boehncke, G. Schumacher and G. Bendas, Biochem. Pharmacol., 2006, 72, 474-485.

37 J. Dernedde, A. Rausch, M. Weinhart, S. Enders, R. Tauber, K. Licha, M. Schirner, U. Zügel, A. von Bonin and R. Haag, Proc. Natl. Acad. Sci. U. S. A., 2010, 107, 19679-19684.

38 A. Mulder, T. Auletta, A. Sartori, S. Del Ciotto, A. Casnati, R. Ungaro, J. Huskens and D. N. Reinhoudt, J. Am. Chem. Soc., 2004, 126, 6627-6636.

39 A. Perl, A. Gomez-Casado, D. Thompson, H. H. Dam, P. Jonkheijm, D. N. Reinhoudt and J. Huskens, Nat. Chem., 2011, 3, 317-322.

40 J. Huskens, A. Mulder, T. Auletta, C. A. Nijhuis, M. J. W. Ludden and D. N. Reinhoudt, J. Am. Chem. Soc., 2004, 126, 6784-6797.

41 M. Wyszogrodzka and R. Haag, Chem. - Eur. J., 2008, 14, 9202-9214.

42 K. Petkau-Milroy and L. Brunsveld, Eur. J. Org. Chem., 2013, 3470-3476.

43 B. Bertolotti, I. Sutkeviciute, M. Ambrosini, R. RibeiroViana, J. Rojo, F. Fieschi, H. Dvorakova, M. Kasakova, K. Parkan, M. Hlavackova, K. Novakova and J. Moravcova, Org. Biomol. Chem., 2017, 15, 3995-4004.

44 G. Goti, A. Palmioli, M. Stravalaci, S. Sattin, M.-G. De Simoni, M. Gobbi and A. Bernardi, Chem. - Eur. J., 2016, 22, 3686.

45 I. Morbioli, V. Porkolab, A. Magini, A. Casnati, F. Fieschi and F. Sansone, Carbohydr. Res., 2017, 453-454, 36-43.

46 D. Diwan, K. Shinkai, T. Tetsuka, B. Cao, H. Arai, T. Koyama, K. Hatano and K. Matsuoka, Molecules, 2017, 22, 157.

47 M. Jerabek-Willemsen, C. J. Wienken, D. Braun, P. Baaske and S. Duhr, Assay Drug Dev. Technol., 2011, 9, 342-353.

48 S. Duhr and D. Braun, Proc. Natl. Acad. Sci. U. S. A., 2006, 103, 19678-19682.

49 S. A. I. Seidel, C. J. Wienken, S. Geissler, M. JerabekWillemsen, S. Duhr, A. Reiter, D. Trauner, D. Braun and P. Baaske, Angew. Chem., Int. Ed., 2012, 51, 10656-10659.

50 S. D. Fihn, N. Engl. J. Med., 2003, 349, 259-266.

51 R. H. Mak and H. J. Kuo, Curr. Opin. Pediatr., 2006, 18, 148-152. 52 D. F. Sahm, C. Thornsberry, D. C. Mayfield, M. E. Jones and J. A. Karlowsky, Antimicrob. Agents Chemother., 2001, 45, 1402-1406.

53 M. Kanai, K. H. Mortell and L. L. Kiessling, J. Am. Chem. Soc., 1997, 119, 9931-9932.

54 D. M. Crothers and H. Metzger, Immunochemistry, 1972, 9, 341-357. 